\title{
Feeding during High-Flow Nasal Cannula for Bronchiolitis: Associations with Time to Discharge
}

\author{
Kristin A Shadman, MD*, Michelle M Kelly, MD, M Bruce Edmonson, MD, MPH, Daniel J Sklansky, MD, \\ Kirstin Nackers, MD, Ann Allen, MD, Christina B Barreda, MD, Anne S Thurber, MS, Ryan J Coller MD, MPH
}

Department of Pediatrics, University of Wisconsin School of Medicine and Public Health, Madison, Wisconsin.

BACKGROUND: High-flow nasal cannula (HFNC) is increasingly used to treat children hospitalized with bronchiolitis; however, the best practices for feeding during HFNC and the impact of feeding on time to discharge and adverse events are unknown. The study objective was to assess whether feeding exposure during HFNC was associated with time to discharge or feedingrelated adverse events.

METHODS: This retrospective cohort study included inpatients aged 1-24 months receiving HFNC for bronchiolitis at an academic children's hospital from January 1, 2015 to March 1, 2017. Feeding exposures during HFNC were categorized as fed or not fed. Among fed children, we further evaluated mixed (oral and tube) or exclusive oral feeding. The primary outcome was time to discharge after HFNC cessation. Secondary outcomes were aspiration, intubation after HFNC, and seven-day readmission.

RESULTS: Of 123 children treated with HFNC, 45 (37 \%) were never fed. A total of 78 children (63\%) we fed; 50 (41\%) were exclusively orally fed and $28(23 \%)$ had mixed feeding. Median (interquartile range) time to discharge after HFNC was 29.5 hours (23.5-47.9) and 39.8 hours (26.4$61.5)$ hours in the fed and not fed groups, respectively. In adjusted models, time to discharge was shorter with any feeding (hazard ratio [HR] 2.17; 95\% Cl: 1.34-3.50) and with exclusive oral feeding (HR 2.13; 95\% Cl: 1.31-3.45) compared with no feeding. Time to discharge from HFNC initiation was shorter for exclusive oral feeding versus not feeding (propensity weighted HR 1.97 [95\% Cl: 1.13-3.43]). Adverse events (one intubation, one aspiration pneumonia, one readmission) occurred in both groups.

LIMITATIONS: Assessment of feeding exposure did not account for quantity and duration.

DISCUSSION: Children fed while receiving HFNC for bronchiolitis may have shorter time to discharge than those not fed. Feeding-related adverse events were rare regardless of the feeding method. Controlled prospective studies addressing residual confounding are needed to justify a change in the current practice. Journal of Hospital Medicine 2019;14:e43-e48. (c) 2019 Society of Hospital Medicine ronchiolitis is the most common cause of nonbirth hospitalization in children in the United States less than one year of age. ${ }^{1}$ For children with severe bronchiolitis, high-flow nasal cannula (HFNC) is increasingly used ${ }^{2-4}$ to reduce work of breathing and prevent the need for further escalation of ventilatory support. ${ }^{5,6}$ Although previous studies suggest that enteral feeding is recommended in the management of patients hospitalized with bronchiolitis, ${ }^{7-9}$ limited evidence exists to guide feeding practices for patients receiving HFNC support. 5,10,11

Respiratory support with HFNC has been associated with prolonged periods without enteral hydration/nutrition (ie, nil per os [NPO] $)^{12}$ primarily due to anticipation of further escalation of respiratory support or concern for increased risk of aspiration. The majority of patients with bronchiolitis managed

*Corresponding Author: Kristin A. Shadman, MD; E-mail: kshadman@ pediatrics.wisc.edu; Telephone: 608-265-8561.

Find Additional Supporting Information in the online version of this article.

Received: April 10, 2019; Revised: August 9, 2019; Accepted: August 12, 2019

(C) 2019 Society of Hospital Medicine DOI 10.12788/jhm.3306 with HFNC, however, do not require escalation of care., ${ }^{5,13}$ When feeding is attempted during HFNC support, it is frequently interrupted. ${ }^{5}$ Moreover, keeping all children NPO when receiving HFNC may be associated with weight loss and longer length of stay (LOS). ${ }^{12,14}$ Two small studies found that children admitted to the intensive care unit who received HFNC support for bronchiolitis did not have increased rates of emesis, worsening respiratory distress or aspiration pneumonia when enterally fed. ${ }^{10,11}$ However, no comparison of adverse events or LOS has been made between patients who were fed and those who were not fed during HFNC therapy, and previous studies have included only patients who have received HFNC in the intensive care setting.

Supporting safe feeding early in hospitalizations for bronchiolitis may facilitate expedited clinical improvement and discharge. As part of an ongoing bronchiolitis quality improvement initiative at our hospital, we sought to characterize feeding practices during HFNC therapy and assess whether feeding exposure was associated with (1) time to discharge after HFNC or (2) feeding-related adverse events. We hypothesized that feeding during HFNC therapy would be associated with a shorter time to discharge after HFNC cessation. 


\section{METHODS}

\section{Study Design, Setting, Participants}

This was a retrospective cohort study of patients aged 1-24 months receiving HFNC support for respiratory failure due to bronchiolitis at an academic children's hospital between January 1, 2015 and March 1, 2017. Our institution has had a clinical practice guideline, associated order set, and respiratory therapy protocol for general care patients with bronchiolitis since 2009. Patients with bronchiolitis who were weaning HFNC have been cared for in both the intensive and general care settings since 2013. A formal process for initiation of HFNC on general care units was instituted in January of 2017. During the study period, no patients with HFNC support for bronchiolitis had all their care entirely outside the intensive care unit at our institution. However, initiation and subsequent use of HFNC may have occurred in either the intensive care or general care setting. No specific guidance for feeding during HFNC existed during this period.

Patients were identified using the Virtual PICU Systems database, (VPS LLC, myvps.org, Los Angeles, California) and, by definition, all patients received at least some of their care in the intensive care unit. Patients with comorbid conditions of prematurity ( $<35$ weeks) and those with cardiopulmonary, neuromuscular, and genetic diseases were included. Patients with preexisting dysphagia, defined as ongoing outpatient speech therapy for swallowing concerns, an admission diagnosis of aspiration pneumonia or on home respiratory support, were excluded. Children $(n=7)$ were excluded if they had more than one period of HFNC during admission. This study was determined to be exempt by the University of Wisconsin School of Medicine and Public Health's Institutional Review Board.

\section{Data Collection and Study Variables}

The following variables were collected from VPS administrative data: patient gender, age, admission and discharge date and time, type and total hours of respiratory support, intensive care admission, and LOS (in hours). Additional demographic, clinical, and feeding exposure variables were abstracted manually from the electronic medical record (Epic, Verona, Wisconsin) using a structured data collection tool and stored in REDCap (Research Electronic Data Capture) ${ }^{15}$ including prematurity, race/ethnicity, insurance status, primary language, and passive tobacco smoke exposure. Clinical variables included duration of illness (days) at the time of admission, unit of HFNC initiation (emergency department, general care, intensive care, respiratory rate and oxygen saturation at HFNC initiation (<90\%, 91\%-92\%, or $>92 \%)$, acquisition of blood gas at HFNC admission, duration of time on HFNC (hours) and need for intubation or noninvasive ventilation prior to HFNC. The Pediatric Index of Mortality 2 Risk of Mortality (PIM 2 ROM) ${ }^{16}$ was used to estimate the severity of illness. The PIM2ROM uses clinical variables (systolic blood pressure, fixed pupils, measure of hypoxia using $\mathrm{PaO}_{2} / \mathrm{FiO}_{2}$ ratio, base excess, mechanical ventilation, elective admission, recovery from surgery, cardiac bypass, high-risk diagnosis, low-risk diagnosis) collected at the time of intensive care admission to generate a score that predicts the risk of mortality for an individual patient. ${ }^{17}$

Feeding exposures were documented in three-hour intervals from HFNC initiation to completion using a structured protocol. At each interval the following feeding information was abstracted from a review of nursing and physician documentation and relevant clinical flowsheets: presence or absence of feeding during the interval, route of feeding (oral, nasogastric [NG] or nasojejunal [NJ]). Feeding exposure was categorized a priori as fed at any point during HFNC (vs not fed at any point). Fed children were further characterized as (1) mixed feeding consisting of oral and tube feeds (NG or NJ) or (2) exclusive oral feeding throughout HFNC support (Appendix 1).

The primary outcome was the number of hours to discharge from HFNC cessation. Secondary outcomes were time to discharge from HFNC initiation, all-cause readmissions within seven days of discharge, and potential feeding-related adverse events. Potential adverse events included: (1) aspiration, defined as initiation of antibiotic AND either chest radiograph official interpreted as evidence for aspiration and/or documented concern for aspiration from the treating physician, or (2) intubation after feeding during HFNC.

\section{Statistical Analysis}

Descriptive statistics evaluated differences in demographics and clinical variables for feeding exposure groups. We used chi-squared tests for differences in proportions and t-tests or Wilcoxon Rank-Sum tests for differences in means or medians for continuous variables, respectively. Associations between feeding exposure during $\mathrm{HFNC}$ and time to discharge (measured in hours) after HFNC completion were modeled with Cox proportional hazards regression. Using this approach, hazard ratios $(H R)>1$ indicate a higher hazard (rate) of discharge for children with a feeding exposure than for children without the exposure. For example, a hazard ratio equal to two indicates that the exposed population is discharged at twice the rate per unit time as the nonexposed population. Death or censoring events did not occur. Feeding exposure was first modeled dichotomously as not fed or fed. To further explore associations between feeding modality and our outcome, we then modeled feeding exposure categorically as not fed (reference), mixed (oral and tube) feeding, or exclusive oral feeding throughout HFNC.

After constructing a set of unadjusted models, we then adjusted the models for variables having independent (bivariate $P<.10$ ) associations with time to discharge: age, unit of HFNC initiation, highest respiratory support required before HFNC, and HFNC duration. Finally, to attempt to account for residual confounding from latent constructs, we also created a set of propensity-weighted Cox proportional hazards models. Propensity weights ${ }^{18}$ reflecting the probability of being fed or never being fed during HFNC were created using logistic regression with predictors we hypothesized a priori that may have influenced the clinical decision to feed during HFNC: age, day of illness on admission, prematurity, PIM2 ROM score, respiratory rate, oxygen saturation and blood gas acquisition at HFNC initiation, and highest respiratory support required 


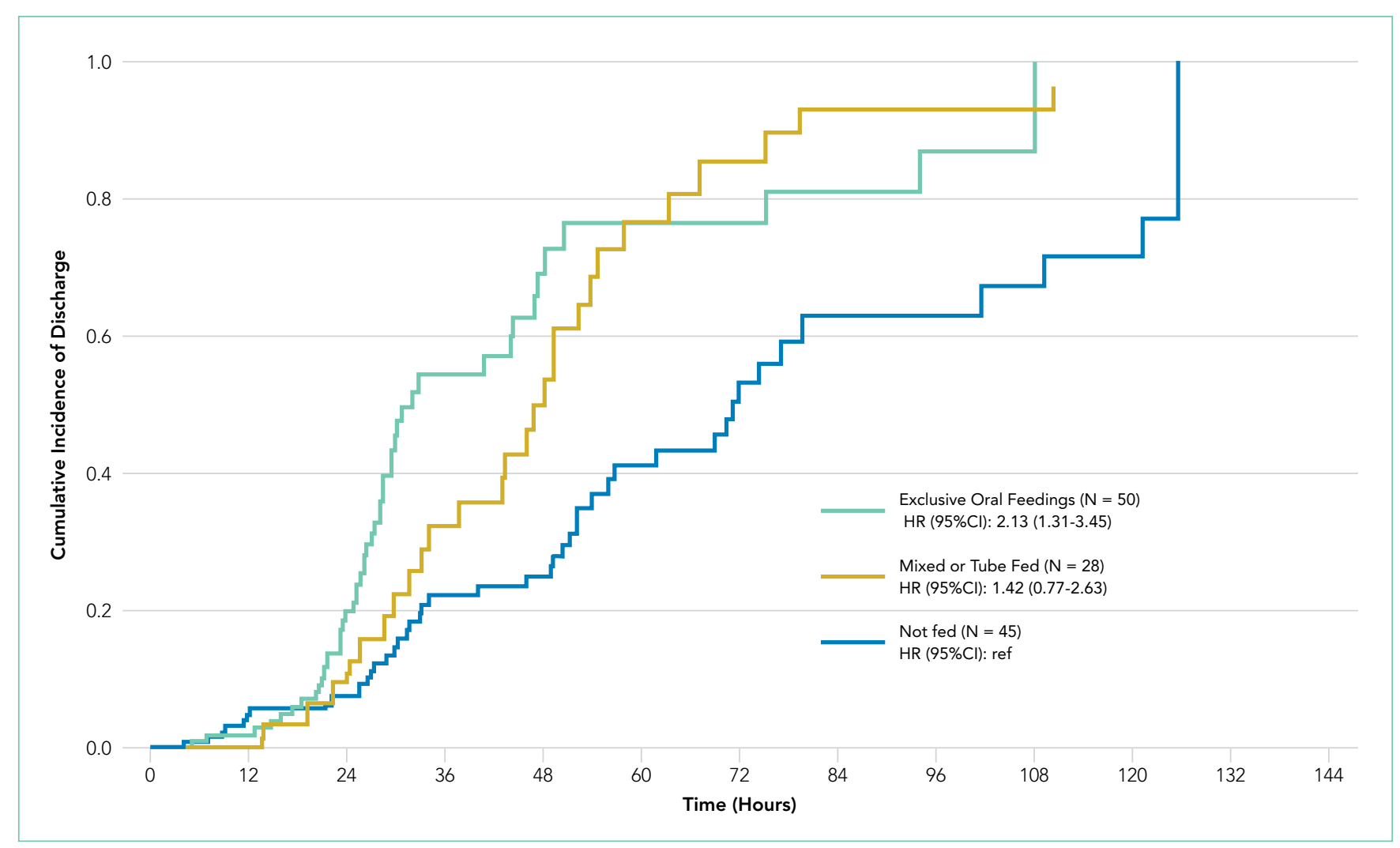

FIG. Adjusted Hazard Ratio for Time to Discharge after Completion of High Flow Nasal Cannula Support According to Feeding Modality

before HFNC. All analyses were conducted using STATA 14.0 (StataCorp, College Station, Texas), and adjusted hazard ratios (aHR) with $95 \%$ confidence intervals $(95 \% \mathrm{Cls})$ were reported.

\section{RESULTS}

Patients $(n=123)$ had a mean age of 7.3 months (standard deviation [SD] 7.1) and presented on day of illness 4.8 (SD 2.3). Prior to HFNC, $10 \%$ required higher respiratory support $(3 \%$ mechanical ventilation). Former preterm children were $12 \%$ of the overall sample.

During HFNC, $37 \%$ of patients were never fed, $41 \%$ were exclusively orally fed, and $23 \%$ had tube or mixed oral and tube feedings (Table 1 and Appendix 2). Children who were not fed were older, but groups were otherwise similar in terms of gender, race/ethnicity, passive smoke exposure, day of illness, unit of HFNC initiation, respiratory support required prior to HFNC, and respiratory rate at HFNC initiation.

Median time to discharge after HFNC completion was 31.4 hours (interquartile range $[I Q R] 23.9-52)$. Median (IOR) time to discharge was 29.5 (IQR 23.5-47.9) hours in children who were fed and 39.8 (26.4-61.5) hours in those who were not fed (unadjusted HR 1.25 [0.86-1.82], aHR 1.83 [95\% Cl: 1.16-2.88]). Time to discharge was shortest when children were fed exclusively orally (Figure). Compared with children who were not fed, time to discharge following HFNC completion was significantly shorter for those who were exclusively orally fed (aHR 2.13 [95\% Cl: 1.31-3.45]; Table 2). Results of the propensity-weighted model were similar: time to discharge after completing
HFNC was shorter in fed versus not fed children (HR 2.17; $95 \%$ $\mathrm{Cl}$ : 1.34-3.50). The secondary outcome, time to discharge from HFNC initiation, had a similar relationship, ie, shorter time to discharge with exclusive oral feeding vs not feeding [aHR 1.95 (95\% Cl: 1.19-3.18)]. Time to discharge after initiation of HFNC was also shorter for fed versus not fed in propensity-weighted analysis (HR 1.97; 95\% Cl: 1.13-3.43).

Adverse events were rare. One otherwise healthy, full-term one-month-old (unfed) child was intubated; one otherwise healthy, full-term four-month-old (fed) infant intubated prior to HFNC therapy had antibiotic initiation with radiologist documentation of possible pneumonia and physician documentation of suspected aspiration pneumonia, and one otherwise healthy, full-term, four-month-old (fed) child had a readmission within seven days.

\section{DISCUSSION}

This observational study found that being fed during HFNC was associated with shorter time to discharge after HFNC support was completed. Exclusive oral feeding was associated with the shortest time to discharge, and these results were consistent across a variety of analytical approaches. Adverse events were rare and occurred in both fed and unfed children.

These findings advance research on relationships between nutrition and bronchiolitis outcomes. Studies of general care patients with bronchiolitis without HFNC have observed associations between poor nutrition and prolonged LOS. 19,20 Two previous studies of patients receiving HFNC therapy for 
TABLE 1. Clinical Characteristics of Children According to Feeding Status during HFNC Therapy

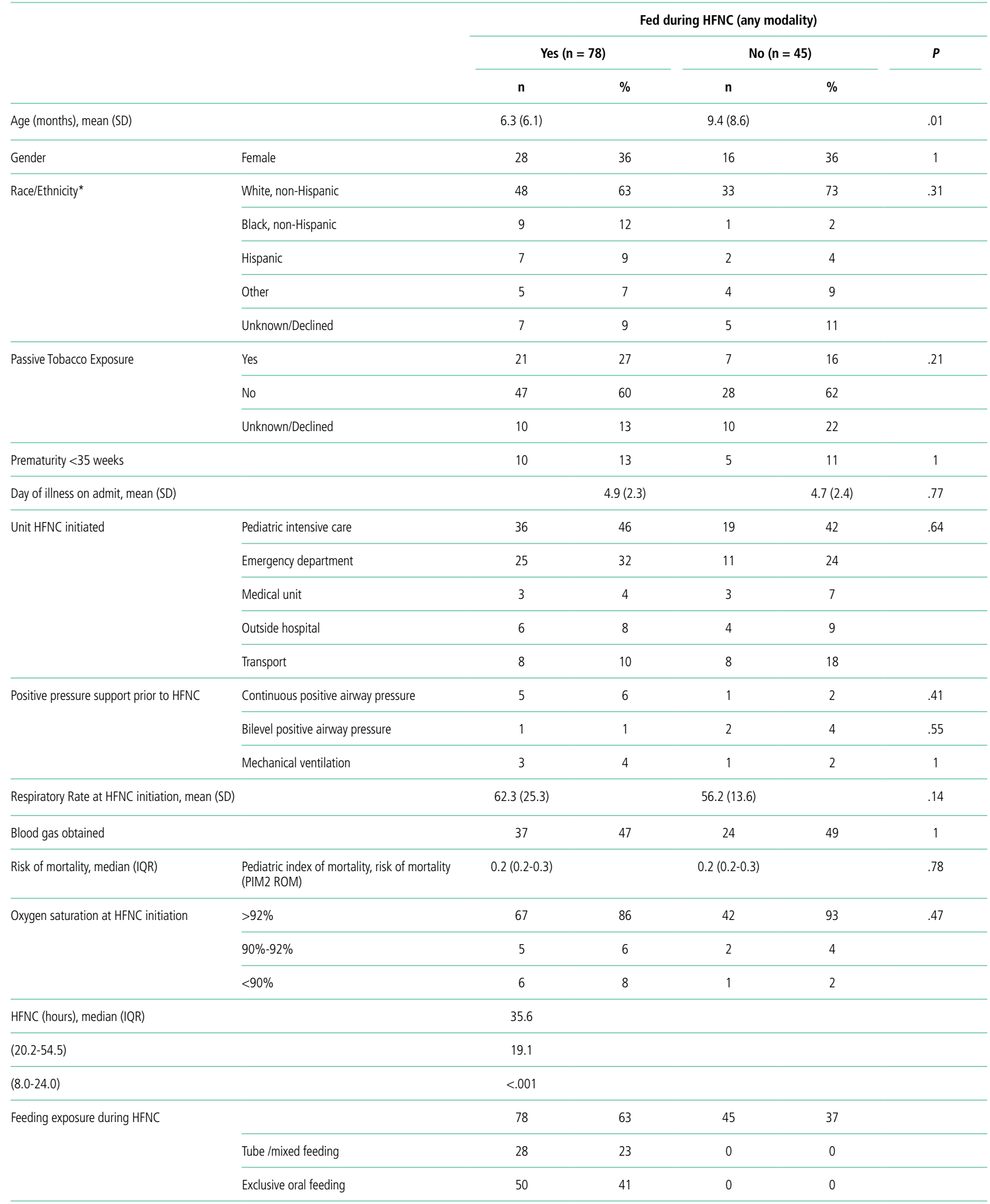

Abbreviations: HFNC, high-flow nasal cannula; IQR, interquartile range; SD, standard deviation. 
TABLE 2. Hazard Ratios for Time to Discharge from Completion of HFNC and Time to Discharge from Initiation of HFNC

\begin{tabular}{|c|c|c|}
\hline & $\begin{array}{l}\text { Unadjusted } \\
\text { HR }(95 \% \mathrm{Cl})\end{array}$ & $\begin{array}{l}\text { Adjusted } \\
\text { HR }(95 \% \mathrm{Cl})^{\mathrm{a}}\end{array}$ \\
\hline \multicolumn{3}{|l|}{ Time to discharge from completion of HFNC by feeding modality during HFNC } \\
\hline Not fed & ref & ref \\
\hline Mixed or tube fed & $0.94(0.59-1.51)$ & $1.42(0.77-2.63)$ \\
\hline Exclusive oral feeding & $1.57(1.04-2.38)$ & $2.13(1.31-3.45)$ \\
\hline Not fed & ref & ref \\
\hline Mixed or tube fed & $0.42(0.26-0.68)$ & $1.27(0.69-2.36)$ \\
\hline \multirow[t]{3}{*}{ Exclusive oral feeding } & $0.89(0.59-1.34)$ & $1.95(1.19-3.18)$ \\
\hline & Propensity Weight & \\
\hline & HR $(95 \% \mathrm{Cl})^{b}$ & \\
\hline $\begin{array}{l}\text { Time to discharge from initiation of HFNC by fed vs not fed during HFNC } \\
\text { (any modality) }\end{array}$ & $1.97(1.13-3.43)$ & \\
\hline \multicolumn{3}{|c|}{$\begin{array}{l}\text { "Models adjusted for age, unit of HFNC initiation, highest respiratory support required before HFNC, Pediatric Index of Mortality } 2 \text { Risk of Mortality (PIM2 ROM), oxygen saturation at HFNC } \\
\text { initiation, and duration of HFNC }\end{array}$} \\
\hline \multicolumn{3}{|c|}{$\begin{array}{l}\text { bPropensity weights reflected the probability of being fed or never being fed during HFNC using logistic regression based on age, day of illness on admission, history of prematurity, respiratory } \\
\text { rate, oxygen saturation, blood gas acquisition and PIM2 ROM at HFNC initiation, and highest respiratory support required before HFNC. }\end{array}$} \\
\hline Abbreviations: HFNC, high-flow nasal Cannula; HR, hazard ratio. & & \\
\hline
\end{tabular}

bronchiolitis concluded that frequent interruption ${ }^{11}$ and later initiation of enteral nutrition ${ }^{10}$ during ICU stay was associated with longer time to discharge. ${ }^{11}$ To our knowledge, this is the first study of patients with bronchiolitis treated with HFNC in both general care and ICU settings that compared outcomes according to whether children were fed during HFNC therapy. Our results extend previous work demonstrating that delays in feeding may be associated with longer LOS.

Decisions to feed children with respiratory distress due to bronchiolitis are complex and often subjective. Readiness to feed may be based upon the assessment of a child's work of breathing, trajectory of illness, institutional culture, and individual physician, nurse, respiratory therapist or speech-language pathologist comfort. In the absence of established feeding best practices, ${ }^{21}$ some institutions have developed guidelines based on local expert opinion; however, often these recommendations remain largely subjective and nonspecific. 5, 10,22-24 Although decisions to feed may be influenced by concern about a child's clinical stability and feeding risk, we found few objective clinical differences between children fed (orally or by enteral tube) or not fed. Moreover, our results were consistent even when we used a propensity-weighted model to account for measured factors that may have been associated with the decision to initiate feeding. This suggests the decision to feed could be more arbitrary than we assume and is important to investigate in future research.

Additionally, although a few early studies have aimed to standardize the process of weaning HFNC support in bronchi- olitis, ${ }^{25,26}$ this process is also largely subjective. ${ }^{10,22,23}$ As such, the weaning process may be influenced by perceptions of the child's overall health. Orally fed children may be viewed as more comfortable or well and thus, more readily weaned, which ultimately influences the length of HFNC therapy. Our study design attempted to account for this potential bias by measuring time to discharge following HFNC therapy, rather than measuring total LOS. Meeting adequate calorie, weight, or hydration goals prior to discharge may take longer if feeds have been withheld. We speculate that prolonged periods of NPO might also risk transient oral aversion or feeding discoordination that could influence LOS. Previous research involving broad intensive care unit populations has established the importance of providing nutrition to critically ill children as soon as possible as a means of improving outcomes. ${ }^{27-29}$ Patients receiving HFNC support for bronchiolitis could plausibly experience similar benefits.

This single-center study with a relatively small sample size has important limitations to consider. The observational design limits our ability to draw conclusions about causal relationships between feeding, time to discharge, and adverse events. In particular, feeding exposure did not account for nuances in feeding timing, feeding density, and other elements of feeding exposure. Additionally, adverse events are rare, and this study is inadequately powered to detect differences between exposure groups. Although we included children cared for in general and intensive care units, our findings may not be generalizable to other hospitals with different placement criteria. Despite the 
creation of adjusted and propensity-weighted models, our results are still subject to possible residual indication bias. We cannot control for all possible confounders, particularly unmeasured factors which might simultaneously motivate decisions whether, when, and how to feed children receiving HFNC therapy and influence time to discharge after HFNC is finished. Although this study observed associations between feeding during HFNC and both our primary (time to discharge after HFNC was complete) and secondary (time to discharge after HFNC was initiated) outcomes, future work should evaluate how feeding strategies might impact total LOS, particularly as management becomes more standardized.

Prospective studies of feeding exposures during HFNC therapy in bronchiolitis, as well as rigorous interventional study designs, are needed to confirm shorter lengths of stay and safety with larger and more diverse samples. Future research should evaluate methods to safely and effectively feed children with severe bronchiolitis, which would inform standardized evidence-based approaches. Given the scale on which children with bronchiolitis are admitted each year, the implications of such work could be substantial.

\section{CONCLUSION}

Children fed while receiving HFNC for bronchiolitis may have shorter time to discharge than those who are not fed. Feeding-related adverse events were rare regardless of the feeding method. Controlled prospective studies addressing residual confounding are needed to justify a change in the current practice.

\section{Acknowledgments}

The authors would like to acknowledge the valuable feedback on earlier drafts from members of the University of Wisconsin Division of Pediatric Hospital Medicine CREATE writing group.

Disclosures: The authors have no conflicts of interest relevant to this article to disclose.

Funding: The authors have no financial relationships relevant to this article to disclose.

\section{References}

1. HCUPnet. https://hcupnet.ahrq.gov/. Accessed February 7, 2019

2. Beggs $\mathrm{S}$, Wong ZH, Kaul S, Ogden KJ, Walters JA. High-flow nasal cannula therapy for infants with bronchiolitis. Cochrane Database Syst Rev. 2014;1(1):CD009609. https://doi.org/10.1002/14651858.CD009609.pub2.

3. Mayfield S, Bogossian F, O'Malley L, Schibler A. High-flow nasal cannula oxygen therapy for infants with bronchiolitis: pilot study. J Paediatr Child Health. 2014;50(5):373-378. https://doi.org/10.1111/jpc.12509.

4. Hilliard TN, Archer N, Laura $\mathrm{H}$, et al. Pilot study of vapotherm oxygen delivery in moderately severe bronchiolitis. Arch Dis Child. 2012;97(2):182-183. https://doi.org/10.1136/archdischild-2011-301151.

5. Franklin $\mathrm{D}, \mathrm{Bab}$ FE, Schlapbach LJ, et al. A randomized trial of high-flow oxygen therapy in infants with bronchiolitis. N Engl J Med. 2018;378(12):1121 1131. https://doi.org/10.1056/NEJMoa1714855

6. McKiernan C, Chua LC, Visintainer PF, Allen H. High flow nasal cannulae therapy in infants with bronchiolitis. J Pediatr. 2010;156(4):634-638. https://doi. org/10.1016/j.jpeds.2009.10.039.
7. Maffey A, Moviglia T, Mirabello C, et al. Swallowing and respiratory distress in hospitalized patients with bronchiolitis. Dysphagia. 2013;28(4):582-587. https://doi.org/10.1007/s00455-013-9470-0.

8. Kugelman A, Raibin $\mathrm{K}$, Dabbah $\mathrm{H}$, et al. Intravenous fluids versus gastric-tube feeding in hospitalized infants with viral bronchiolitis: a randomized, prospective pilot study. J Pediatr. 2013;162(3):640-642.e641. https://doi. org/10.1016/j.jpeds.2012.10.057.

9. Oakley E, Borland M, Neutze J, et al. Nasogastric hydration versus intravenous hydration for infants with bronchiolitis: a randomised trial. Lancet Respir Med. 2013;1(2):113-120. https://doi.org/10.1016/S2213-2600(12)70053-X.

10. Slain KN, Martinez-Schlurmann N, Shein SL, Stormorken A. Nutrition and high-flow nasal cannula respiratory support in children with bronchiolitis. Hosp Pediatr. 2017;7(5):256-262. https://doi.org/10.1542/hpeds.2016-0194.

11. Sochet AA, McGee JA, October TW. Oral nutrition in children with bronchiolitis on high-flow nasal cannula is well tolerated. Hosp Pediatr. 2017;7(5):249255. https://doi.org/10.1542/hpeds.2016-0131.

12. Canarie MF, Barry S, Carroll CL, et al. Risk factors for delayed enteral nutrition in critically ill children. Pediatr Crit Care Med. 2015;16(8):e283-e289. https:// doi.org/10.1097/PCC.0000000000000527.

13. Schibler A, Pham TM, Dunster KR, et al. Reduced intubation rates for infants after introduction of high-flow nasal prong oxygen delivery. Intensive Care Med. 2011;37(5):847-852. https://doi.org/10.1007/s00134-011-2177-5.

14. Hamilton S, McAleer DM, Ariagno K, et al. A stepwise enteral nutrition algorithm for critically ill children helps achieve nutrient delivery goals*. Pediatr Crit Care Med. 2014;15(7):583-589. https://doi.org/10.1097/ PCC. 0000000000000179

15. Harris PA, Taylor R, Thielke R, et al. Research electronic data capture (REDCap)--a metadata-driven methodology and workflow process for providing translational research informatics support. J Biomed Inform. 2009;42(2):377381. https://doi.org/10.1016/j.jbi.2008.08.010.

16. Slater A, Shann F, Group APS. The suitability of the Pediatric Index of Mortality (PIM), PIM2, the Pediatric Risk of Mortality (PRISM), and PRISM III for monitoring the quality of pediatric intensive care in Australia and New Zealand. Pediatr Crit Care Med. 2004;5(5):447-454. https://doi.org/10.1097/01. PCC.0000138557.31831.65.

17. Slater A, Shann F, Pearson G, Paediatric Index of Mortality Study G. PIM2: a revised version of the Paediatric Index of Mortality. Intensive Care Med. 2003;29(2):278-285. https://doi.org/10.1007/s00134-002-1601-2.

18. Lanza ST, Moore JE, Butera NM. Drawing causal inferences using propensity scores: a practical guide for community psychologists. Am J Commun Psychol. 2013;52(3-4):380-392. https://doi.org/10.1007/s10464-013-9604-4.

19. Weisgerber MC, Lye PS, Li SH, et al. Factors predicting prolonged hospital stay for infants with bronchiolitis. J Hosp Med. 2011;6(5):264-270. https://doi. org/10.1002/jhm.903.

20. Halvorson EE, Chandler N, Neiberg R, Ervin SE. Association of NPO status and type of nutritional support on weight and length of stay in infants hospitalized with bronchiolitis. Hosp Pediatr. 2013;3(4):366-370. https://doi. org/10.1542/hpeds.2013-0011.

21. Ralston SL, Lieberthal AS, Meissner HC, et al. Clinical practice guideline: the diagnosis, management, and prevention of bronchiolitis. Pediatrics. 2014;134(5):e1474-e1502. https://doi.org/10.1542/peds.2014-2742.

22. Seattle Children's Hospital ZS, Beardsley E, Crotwell D, et al. Bronchiolitis Pathway. http:// www.seattlechildrens.org/pdf/bronchiolitis-pathway.pdf. Accessed January 29, 2019

23. Children's Hospital of Philidelphia DM, Zorc J, Kreindler, J, et al. Inpatient Pathway for Treatment of the Child with Bronchiolitis. https://www.chop. edu/clinical-pathway/bronchiolitis-inpatient-treatment-clinical-pathway. Accessed January 29, 2019

24. Children's Hospital Colorado TA, Topoz I, Freeman J, et al. Pediatric Viral Bronchiolitis. https://www.childrenscolorado.org/globalassets/healthcare-professionals/clinical-pathways/bronchiolitis.pdf. Accessed January 29, 2019

25. Betters KA, Hebbar KB, McCracken C, et al. A novel weaning protocol for highflow nasal cannula in the PICU. Pediatr Crit Care Med. 2017;18(7):e274-e280. https://doi.org/10.1097/PCC.0000000000001181.

26. Kepreotes E, Whitehead B, Attia J, et al. High-flow warm humidified oxygen versus standard low-flow nasal cannula oxygen for moderate bronchiolitis (HFWHO RCT): an open, phase 4, randomised controlled trial. Lancet. 2017;389(10072):930-939. https://doi.org/10.1016/S0140-6736(17)30061-2. 